\title{
DIAGNÓSTICOS DE ENFERMAGEM IDENTIFICADOS EM CRIANÇAS COM INFECÇÃO RESPIRATÓRIA AGUDA
}

\author{
NURSING DIAGNOSES IDENTIFIED IN CHILDREN WITH ACUTE RESPIRATION INFECTION
}

\author{
DIAGNÓSTICOS ENFERMEROS IDENTIFICADOS EN NIÑOS CON INFECCIÓN RESPIRATORIA AGUDA
}

\section{Flávia Paula Magalhães Monteiro ${ }^{1}$, Viviane Martins da Silva ${ }^{2}$, Marcos Venícios de Oliveira Lopes $^{3}$}

RESUMO: Estudo transversal desenvolvido com 78 crianças com até cinco anos de idade, acometidas por infecções respiratórias agudas internadas em um hospital pediátrico de uma capital da região nordeste, com o objetivo de identificar os diagnósticos de enfermagem apresentados por estas crianças. $O$ número de diagnósticos de enfermagem, características definidoras, fatores relacionados e fatores de risco identificados e as demais variáveis numéricas foram analisadas com base em suas medidas de tendência central e dispersão. Foram identificados um total de 26 diagnósticos de enfermagem, 43 fatores relacionados, 14 fatores de risco e 67 características definidoras diferentes. Em média, foram encontrados 5,32 diagnósticos de enfermagem; 4,10 fatores relacionados; 2,03 fatores de risco e 7,33 características definidoras. Os diagnósticos de enfermagem com maior proporção foram: padrão respiratório ineficaz, risco para crescimento desproporcional, proteção ineficaz e mucosa oral prejudicada. Conclui-se que crianças com infecções respiratórias agudas apresentam um quadro diagnóstico complexo incluindo respostas humanas de múltiplos domínios.

PALAVRAS CHAVE: Infecção; Diagnóstico de enfermagem; Criança.

ABSTRACT: A cross-sectional study developed with 78 children with until five years old, bearers of acute respiration infection interned in pediatric hospital of the periphery of a great city, with the purpose to identify the nursing diagnoses presented by these children. The number of nursing diagnoses, defining characteristics, related factors and risk factors identified and other numerical variables were analyzed based in theirs central tendency and dispersion measures. It was identified a total of 26 nursing diagnoses, 43 related factors, 14 risk factors $e$ 67 defining characteristics. In average, It was found 5,32 nursing diagnoses; 4,10 related factors; 2,03 risk factors and 7,33 defining characteristics. The nursing diagnoses with the biggest proportion were: Ineffective Breathing Pattern, Risk for delayed growth, Ineffective protection and Altered oral mucous membrane. We concluded that children with acute respiration infection present a complex diagnostic frame including human responses of multiples domains.

KEY WORDS: Infection; Nursing diagnoses; Child.
RESUMEN: Estudio transversal desarrollado con 78 niños con hasta cinco años de edad, y diagnostico de infección respiratoria aguda ingresadas en un hospital pediátrico de la periferia de una gran ciudad, con el objetivo de identificar los diagnósticos enfermeros presentados por estos niños. El número de diagnósticos enfermeros, características definitorias, factores relacionados y factores de riesgo identificados y las demás variables numéricas fueron analizadas con base en sus medidas de tendencia central y dispersión. Fueron identificados un total de 26 diagnósticos enfermeros, 43 factores relacionados, 14 factores de riesgo y 67 características definitorias diferentes. En media, fueron encontrados 5,32 diagnósticos enfermeros; 4,10 factores relacionados; 2,03 factores de riesgo y 7,33 características definitorias. Los diagnósticos enfermeros con mayor proporción fueron: patrón respiratorio ineficaz, riesgo de crecimiento desproporcionado, protección inefectiva y deterioro de la mucosa oral. Se concluye que niños con infecciones respiratorias agudas presentan un cuadro diagnóstico complejo incluyendo respuestas humanas de múltiples dominios.

PALABRAS CLAVE: Infección; Diagnóstico de enfermería; Niño.

\footnotetext{
${ }^{1}$ Acadêmica de Enfermagem do $8^{\circ}$ Semestre. Bolsista de Extensão / UFC. Endereço: Rua: Raul Pompéia, no 12, Carlito Pamplona,. Cep: 60335-420. Fortaleza- CE. E-mail: flaviapmm@yahoo.com.br ${ }^{2}$ Enfermeira. Professora da Faculdade Católica Rainha do Sertão. Aluna do Curso de Doutorado em Enfermagem da UFC. Fortaleza/CE. E-mail: vivianemartinsdasilva@hotmail.com

${ }^{3}$ Doutor em Enfermagem. Professor Adjunto do Departamento de Enfermagem da Universidade Federal de Ceará./ UFC. Fortaleza/CE. E-mail: marcos@ufc.br
} 


\section{INTRODUÇÃO}

As infecções respiratórias agudas (IRAs) representam doenças de diversas etiologias e grau de complexidade que atingem crianças nos diferentes períodos do ano. Constituem uma das principais causas de morbi-mortalidade em todo o mundo (RODRIGUES et al, 2004), sendo as pneumonias responsáveis por cerca de quatro milhões de óbitos por ano nos países em desenvolvimento (FERREIRA \& BRITTO, 2003). A incidência das IRAs é semelhante em todo o mundo, tanto em países desenvolvidos, como em países em vias de desenvolvimento, diferenciando-se na freqüência e gravidade dos casos (BOTELHO et al, 2003).

As IRAs são problemas respiratórios que acometem crianças, principalmente nos primeiros cinco anos de vida pela suscetibilidade e imaturidade do trato respiratório nessa faixa etária. Além de sofrer a influência de fatores socioeconômicos, em virtude das desigualdades existentes, as IRAs são causas de grande impacto sobre as taxas de hospitalização em crianças. Isto incorre em alta demanda nos serviços de saúde exigindo uma complexidade de ações, em decorrência da peculiaridade da clientela assistida que, ainda em idade precoce, sofrem junto aos familiares, os processos traumáticos da internação hospitalar. $O$ risco de vida e a internação representam, para o familiar, ameaças que variam em conteúdo e intensidade, acarretando medo, angústia e ansiedade (ANDRAUS et al, 2004).

As infecções respiratórias infantis incluem em sua sintomatologia tosse, febre, dispnéia, dor de garganta, dor de ouvido, anorexia, coriza nasal, tiragem sub e intercostal, e cianose (BARATA et al, 1996). Caracterizam-se por crises dependentes ou não da exposição da criança ao agente infeccioso, representadas por várias formas clínicas, dificultando estratégias para o controle ou erradicação dessas infecções. Tomando como base os critérios para definição de caso das IRAs pelo Programa de Assistência e Controle das infecções do Ministério da saúde, a classificação das infecções respiratória agudas baseiam-se na anatomia das vias aéreas, tendo como limite a epiglote, portanto acima da epiglote constituem o grupo de infecções das vias aéreas superiores (IVAS) e as abaixo da epiglote, infecções das vias aéreas inferiores (IVAI) (BRASIL, 1994).

Além disso, inúmeros fatores de risco estão relacionados aos problemas respiratórios em crianças, como idade da criança, $n^{\circ}$ de moradores por domicílio, desnutrição, escolaridade materna, desmame precoce, estação climática, tabagismo passivo, revelando uma maior incidência de infecções em crianças cujos pais apresentam baixa renda familiar, dificuldade de acesso ao serviço de saúde ou até mesmo a precariedade destes serviços, resultando no agravamento da doença (CAETANO et al, 2002).
Estudos desenvolvidos nessa área mostram que elevados índices de pessoas por domicílio estão associados com doença aguda das vias aéreas inferiores na análise bruta, especialmente quando há um expressivo número de pessoas que compartilham - mesmo cômodo da residência com crianças (PRIETSCH et al, 2002). Em decorrência dos altos índices das IRAs no meio infantil serem considerados parâmetros básicos para o estabelecimento das necessidades de saúde desse grupo, tornou-se necessário traçar estratégias para o controle e uma avaliação mais específica e individualizada pelos profissionais de enfermagem através do diagnóstico de enfermagem, onde o enfermeiro poderá atuar interpretando e julgando os problemas de saúde atuais ou potenciais baseados na manifestação das respostas humanas do paciente (LIRA, 2005).

Considerando a escassez de trabalhos sobre diagnósticos de enfermagem nesta clientela que contribuam para o manejo de ações preventivas ou corretivas sobre o problema apontado, sentimos a necessidade de identificar os diagnósticos de enfermagem apresentados por crianças acometidas por IRAs em um hospital pediátrico da cidade de Fortaleza - CE, com vistas a fornecer indicadores para propor intervenções direcionadas e de competência exclusiva do enfermeiro, contribuindo para a elevação da qualidade da prática, através de um modelo de assistência mais eficaz.

\section{MATERIAL E MÉTODOS}

Estudo transversal desenvolvido junto a crianças com diagnóstico de infecção respiratória aguda (IRA). A amostra foi selecionada por conveniência de forma consecutiva na medida em que estas eram internadas. A estimativa do tamanho da amostra foi realizada com base nos dados extraídos no serviço de arquivo médico e estatístico (SAME), e calculada a partir da seguinte fórmula: $n=$ $\left(Z_{\alpha}^{2}\right.$. P.Q .N) / $\left[Z_{\alpha}^{2}\right.$. P.Q $\left.+(N-1) \cdot E^{2}\right]$, onde: $Z_{\alpha}=$ Coeficiente de confiança; $P=$ Prevalência do fenômeno; $Q=$ Complementar de prevalência (1-P); N = População; $\mathrm{E}$ = erro aleatório.

Foram considerados como parâmetros o nível de confiança de $95 \%$ e o erro amostral de $10 \%$, sendo a amostra estabelecida em 78 crianças com até cinco anos de idade. Optamos por trabalhar com crianças nesta faixa etária, pois aproximadamente $50 \%$ dos atendimentos pediátricos correspondem a crianças com até cinco anos portadores de sinais e sintomas decorrentes de algum diagnóstico de IRA (BOTELHO et al, 2003). A prevalência do fenômeno foi estimada em $P=37,75 \%$, através da média das prevalências de crianças internadas com infecção respiratória aguda com base em estudos anteriores (DUARTE \& BOTELHO, 2000; BOTELHO et al, 2003).

O estudo foi realizado em hospital pediátrico de pequeno porte localizado na periferia de uma capital da região nordeste no período de abril a setembro de 
2005. Como instrumento de coleta de dados, foi utilizado um roteiro de avaliação de saúde adaptado de estudo anterior o qual havia sido submetido a avaliação de conteúdo e aparência (SILVA, 2004), o qual foi desenvolvido com base na taxonomia II da North American Nursing Diagnosis Association NANDA dividido em: dados socioeconômicos dos pais/responsáveis e dados referentes às características definidoras, fatores relacionados e informações clínicas complementares e evoluções do estado clínico. Para análise nutricional foram calculados os escores $Z$ de peso por altura, peso por idade e altura por idade.

A coleta de dados ocorreu em dois momentos, primeiramente através de dados primários, mediante exame físico da criança e entrevista de saúde junto aos pais / responsáveis. Os dados secundários foram coletados através de consultas ao prontuário. Posteriormente, foram identificadas as respostas humanas presentes na amostra, bem como os dados referentes às características definidoras e aos fatores relacionados, com 0 propósito de formular os diagnósticos de enfermagem.

O processo de elaboração e inferência dos diagnósticos e problemas colaborativos seguiu as etapas preconizadas pela literatura especializada (GORDON, 1994): coleta, interpretação / agrupamento das informações e nomeação das categorias. Para a denominação dos diagnósticos, utilizou-se a Taxonomia II da NANDA .

No processo de inferência diagnóstica, as histórias clínicas eram individualmente avaliadas pelos autores. Os diagnósticos que apresentavam concordância entre todos foram aceitos. Aqueles em que havia discordância entre os avaliadores, eram reavaliados em suas histórias clínicas até que se obtivesse um consenso.

$O$ estudo foi desenvolvido seguindo os aspectos éticos recomendados pela resolução 196/96 (BRASIL, 1996), sobre pesquisa envolvendo seres humanos, mediante a aprovação do Comitê de Ética local sob o número 30/05, e após a assinatura do termo de consentimento pós-informação. Após a coleta, os dados foram armazenados no Excel e analisados com auxílio do software SPSS 13.0, considerando para a significância estatística um nível de $5 \%$. Os dados nutricionais foram analisados com apoio do módulo NutStat do software Epi Info 3.3, cujo parâmetro adotado foi o escore $Z$ que representa unidades de desvio padrão dentro dos valores que uma população pode assumir, com relação a média que apresenta.

Os dados foram organizados em tabelas com freqüência absolutas e percentuais. O número de diagnósticos de enfermagem, características definidoras, fatores relacionados e fatores de risco identificados e as demais variáveis numéricas foram analisadas com base em suas medidas de tendência central e dispersão.

\section{RESULTADOS}

A maioria das crianças era do sexo masculino $(55,1 \%)$, com idade média de aproximadamente 18 meses $( \pm 12,3)$, sendo que $25 \%$ da amostra apresentaram idade até 7 meses e $75 \%$ idade até 27 meses. O tempo médio de amamentação exclusiva foi de 3,47 meses $( \pm 3,32)$, sendo que $25 \%$ das crianças foram amamentadas até 1 mês e $75 \%$ foram amamentadas durante 4 meses. Quanto à alimentação, $70,5 \%$ da amostra faziam uso de dieta suplementar.

Com relação à procedência e naturalidade, $88,5 \%$ crianças eram da capital. A média da idade das mães foi de 24,95 anos $( \pm 6,09)$, com média de filhos de 2,64 ( $\pm 1,61)$, e tempo médio de escolaridade da mãe de 2,68 anos $( \pm 0,49)$. Os genitores eram, em sua maioria, cuidadores do lar $(64,1 \%)$, com média de $5,46( \pm 2,62)$ pessoas residentes por domicílio e renda familiar entre 0,5 a 1 salário mínimo para $41,0 \%$ dos entrevistados. Quanto ao diagnóstico médico, 74,35\% das crianças apresentaram pneumonia.

As médias das medidas antropométricas das crianças foram: comprimento de $73,90 \mathrm{~cm}( \pm 16,38)$; peso de $10,34 \mathrm{~kg}( \pm 3,07)$; escore $Z$ de peso / altura foi de 0,47 ( $\pm 1,31)$; circunferência abdominal de $48,75 \mathrm{~cm}( \pm 5,13)$; perímetro cefálico de $46,25 \mathrm{~cm}$ $( \pm 3,67)$; perímetro torácico de $48,52 \mathrm{~cm}( \pm 4,89)$.

As crianças avaliadas apresentaram, em média, 5,32 diagnósticos $( \pm 1,79)$, sendo identificadas um total de 67 características definidoras nas crianças com média foi de $7,33( \pm 2,86)$, e 43 fatores relacionados com média de $4,10( \pm 1,94)$. 
Tabela 1 - Distribuição das características definidoras presentes em crianças acometidas por infecção respiratória aguda. Fortaleza, 2005.

\begin{tabular}{|c|c|c|c|}
\hline Características definidoras & $N^{0}$ & $\%$ & \\
\hline 1. Ruídos adventícios respiratórios & 75 & 96,2 & \\
\hline 2. Dispnéia & 58 & 74,4 & \\
\hline 3. Respiração curta & 54 & 69,2 & \\
\hline 4. Tosse ineficaz ou ausente & 47 & 60,3 & \\
\hline 5. Mudança na freqüência e no ritmo respiratório & 46 & 59,0 & \\
\hline 6. Tosse & 28 & 35,9 & \\
\hline 7. expectoração & 26 & 33,3 & \\
\hline 8. Incapacidade para lavar o corpo ou partes do corpo & 26 & 33,3 & \\
\hline 9. Freqüência respiratória /min -ldades de 1-4 anos: $<20$ ou >30 & 25 & 32,1 & \\
\hline 10. Manchas/ placas brancas na mucosa oral & 22 & 28,2 & \\
\hline 11. Uso da musculatura acessória para respirar & 20 & 25,6 & \\
\hline 12. Estímulos considerados uma ameaça & 11 & 14,1 & \\
\hline 13. Palidez & 10 & 12,8 & \\
\hline 14. Escore Z > +2 & 10 & 12,8 & \\
\hline 15. Diâmetro ântero - posterior aumentado & 9 & 11,5 & \\
\hline 16. Sucção/ deglutição no peito regular e sustentada & 7 & 9,0 & P75 \\
\hline 17. Freqüência respiratória/ min - Bebês: $<25$ ou > 60 & 6 & 7,7 & \\
\hline 18. A criança está contente após a mamada & 5 & 6,4 & \\
\hline 19. Pelo menos três evacuações de fezes líquidas por dia & 5 & 6,4 & \\
\hline 20. Descoloração do esmalte do dente & 4 & 5,1 & \\
\hline 21. Sons respiratórios diminuídos & 4 & 5,1 & \\
\hline 22. Esforço para evacuar & 4 & 5,1 & \\
\hline 23. Cárie na coroa do dente ou raiz & 4 & 5,1 & \\
\hline 24. Fezes duras e secas & 3 & 3,8 & \\
\hline 25. Língua geográfica & 3 & 3,8 & \\
\hline 26. Relato de estar assustado & 3 & 3,8 & \\
\hline 27. Três ou mais despertares durante a noite & 3 & 3,8 & \\
\hline 28. Instabilidade postural durante a execução de atividades rotineiras da vida diária & 2 & 2,6 & \\
\hline 29. Capacidade limitada para desempenhar as habilidades motoras grossas & 2 & 2,6 & \\
\hline 30. Capacidade limitada para desempenhar as habilidades motoras finas & 2 & 2,6 & \\
\hline 31. Dilatação pupilar (medo) & 2 & 2,6 & \\
\hline 32. Dilatação nasal & 2 & 2,6 & \\
\hline 33. Rompimento da superfície da pele (epiderme) & 2 & 2,6 & P50 \\
\hline 34. Expressão facial (dor) & 2 & 2,6 & \\
\hline 35. Comportamento expressivo (dor) & 2 & 2,6 & \\
\hline 36. Apetite aumentado & 2 & 2,6 & \\
\hline 37. Relato de nervosismo (medo) & 2 & 2,6 & \\
\hline 38. A mãe é capaz de posicionar a criança no peito para promover uma resposta bem-sucedida & 2 & 2,6 & \\
\hline 39.Comer em resposta a sugestões internas além da fome & 2 & 2,6 & \\
\hline 40. Retardo ou dificuldade em desempenhar habilidades típicas do grupo etário & 2 & 2,6 & \\
\hline 41. Anorexia & 2 & 2,6 & \\
\hline 42. Agitação & 1 & 1,3 & \\
\hline 43. Olhos arregalados & 1 & 1,3 & \\
\hline 44. Despertar mais cedo ou mais tarde do que o desejado & 1 & 1,3 & \\
\hline 45. Mucosas pálidas & 1 & 1,3 & \\
\hline 46. Relato de ingestão inadequada de comida & 1 & 1,3 & \\
\hline 47. Evidência observada (dor) & 1 & 1,3 & \\
\hline 48. Distúrbio do sono (dor) & 1 & 1,3 & \\
\hline 49. Relato verbal ou codificado (dor) & 1 & 1,3 & \\
\hline 50. Dificuldade na fala & 1 & 1,3 & P25 \\
\hline 51. Vesículas & 1 & 1,3 & \\
\hline 52. Dor à evacuação & 1 & 1,3 & \\
\hline 53. Incapacidade de coordenar a sucção, a deglutição e a respiração & 1 & 1,3 & \\
\hline 54. Padrão de comunicação alterado & 1 & 1,3 & \\
\hline 55. Mudança relatada ou medida na acuidade sensorial & 1 & 1,3 & \\
\hline 56. Mudança na resposta usual aos estímulos & 1 & 1,3 & \\
\hline 57. Relato de excitação & 1 & 1,3 & \\
\hline 58. Fadiga & 1 & 1,3 & \\
\hline 59. Aumento na temperatura corporal acima dos parâmetros normais & 1 & 1,3 & \\
\hline 60. Taquicardia & 1 & 1,3 & \\
\hline 61. Calor ao toque & 1 & 1,3 & \\
\hline 62. Fraqueza & 1 & 1,3 & \\
\hline 63. Dor abdominal & 1 & 1,3 & \\
\hline 64. Hiperemia do lábio inferior & 1 & 1,3 & \\
\hline 65. Destruição das camadas da pele (derme) & 1 & 1,3 & \\
\hline 66. Escore $Z<-2$ & 1 & 1,3 & \\
\hline 67. Verbalização materna de satisfação com o processo de amamentação & 1 & 1,3 & \\
\hline
\end{tabular}

P25 - Percentil 25; P50 - Percentil 50; P75 - Percentil 75.

Dezesseis características definidoras percentil 75 e estavam relacionadas ao padrão identificadas apresentaram freqüência acima do respiratório, ao autocuidado, à segurança / proteção, 
e ao desempenho de papel. A maioria das características definidoras se relacionava com alterações respiratórias, encontradas em mais de $25 \%$ das crianças, indicando características peculiares da população em questão. Dentre as características de maior freqüência, estão ruídos adventícios respiratórios (96,2\%), dispnéia (74,4\%), respiração curta $(69,2 \%)$ e tosse ineficaz ou ausente $(60,3 \%) \quad$ (Vide Tabela

Tabela 2 - Distribuição dos diagnósticos de enfermagem presentes em crianças acometidas por infecção respiratória aguda. Fortaleza, 2005.

\begin{tabular}{|c|c|c|c|}
\hline Diagnósticos de enfermagem & $N^{0}$ & $\%$ & \\
\hline 1. Desobstrução ineficaz de vias aéreas & 78 & 100,0 & \\
\hline 2. Risco para infecção & 78 & 100,0 & \\
\hline 3. Padrão respiratório ineficaz & 58 & 74,4 & \\
\hline 4. Risco para crescimento desproporcional & 38 & 48,7 & \\
\hline 5. Proteção ineficaz & 30 & 38,5 & \\
\hline 6. Membrana mucosa oral prejudicada & 27 & 34,6 & P75 \\
\hline 7. Déficit no autocuidado para banho/ higiene & 25 & 32,1 & \\
\hline 8. Medo & 12 & 15,4 & \\
\hline 9. Nutrição desequilibrada: mais do que as necessidades corporais & 10 & 12,8 & \\
\hline 10. Risco para constipação & 10 & 12,8 & \\
\hline 11. Amamentação eficaz & 8 & 10,3 & \\
\hline 12. Dentição prejudicada & 7 & 9,0 & \\
\hline 13. Risco para volume de líquidos deficiente & 5 & 6,4 & P50 \\
\hline 14. Diarréia & 5 & 6,4 & \\
\hline 15. Constipação & 4 & 5,1 & \\
\hline 16. Padrão de sono perturbado & 3 & 3,8 & \\
\hline 17. Integridade da pele prejudicada & 3 & 3,8 & \\
\hline 18. Mobilidade física prejudicada & 2 & 2,6 & \\
\hline 19. Crescimento e desenvolvimento retardados & 2 & 2,6 & \\
\hline 20. Risco para integridade da pele prejudicada & 2 & 2,6 & P25 \\
\hline 21. Dor aguda & 2 & 2,6 & \\
\hline 22. Risco para aspiração & 2 & 2,6 & \\
\hline 23. Nutrição desequilibrada: menos do que as necessidades corporais & $\overline{1}$ & 1,3 & \\
\hline 24. Padrão ineficaz de alimentação infantil & 1 & 1,3 & \\
\hline 25. Percepção sensorial perturbada visual e auditiva & 1 & 1,3 & \\
\hline 26. Hipertermia & 1 & 1,3 & \\
\hline
\end{tabular}

P25 - Percentil 25; P50 - Percentil 50; P75 - Percentil 75.

Seis diagnósticos de enfermagem apresentaram freqüências acima do percentil 75 (Tabela 2). Esses diagnósticos pertenciam as seguintes classes e domínios: desobstrução ineficaz de vias aéreas, proteção ineficaz, mucosa oral prejudicada e risco para infecção ao domínio Segurança / Proteção, sendo que os três primeiros pertencem à classe Lesão Física e o último à classe Infecção; o diagnóstico padrão respiratório ineficaz pertence ao domínio Atividade/ Repouso e à classe Respostas Cardiovasculares/ Pulmonares; 0 diagnóstico risco para crescimento desproporcional pertence ao domínio Crescimento/ Desenvolvimento e à classe Crescimento.

Tabela 3 - Distribuição dos fatores relacionados presentes em crianças acometidas por infecção respiratória aguda. Fortaleza, 2005. 


\begin{tabular}{|c|c|c|c|}
\hline 1. Secreções retidas & 77 & 98,7 & \\
\hline 2. Hiperventilação & 58 & 74,4 & \\
\hline 3. Perfis sanguíneos anormais & 30 & 38,5 & \\
\hline 4. Barreiras ambientais & 26 & 33,3 & \\
\hline 5. Higiene oral ineficaz & 26 & 33,3 & \\
\hline $\begin{array}{l}\text { 6. Separação do sistema de apoio em situação potencialmente estressante } \\
\text { (hospitalização, procedimentos hospitalares) }\end{array}$ & 12 & 15,4 & \\
\hline 7. Ingestão excessiva em relação às necessidades metabólicas & 10 & 12,8 & \\
\hline 8. Imunossupressão & 9 & 11,5 & \\
\hline 9. Asma & 8 & 10,3 & \\
\hline 10. Confiança materna & 7 & 9,0 & P75 \\
\hline 11. Conhecimento básico sobre amamentação & 7 & 9,0 & \\
\hline 12. efeitos adversos de medicações & 4 & 5,1 & \\
\hline 13. Prescrição de algumas medicações & 3 & 3,8 & \\
\hline 14. Respiração pela boca & 3 & 3,8 & \\
\hline 15. Secreções nos brônquios & 3 & 3,8 & \\
\hline 16. Atraso de desenvolvimento & 2 & 2,6 & \\
\hline 17. Infecção & 2 & 2,6 & \\
\hline 18. Responsividade inconsistente & 2 & 2,6 & \\
\hline 19. Hábitos alimentares deficientes & 2 & 2,6 & \\
\hline 20. Agentes biológicos & 2 & 2,6 & \\
\hline 21. Hábitos de evacuação irregulares & 2 & 2,6 & P50 \\
\hline 22. Pele molhada & 2 & 2,6 & \\
\hline 23. Inalação de fumaça & 2 & 2,6 & \\
\hline 24. Parasitas & 2 & 2,6 & \\
\hline 25. Barulho & 1 & 1,3 & \\
\hline 26. Muco excessivo & 1 & 1,3 & \\
\hline 27. Efeitos de incapacidade física & 1 & 1,3 & \\
\hline 28. Padrão de dormir-acordar da mãe & 1 & 1,3 & \\
\hline 29. Despertar causado por outros & 1 & 1,3 & \\
\hline 30. Ingestão de líquidos insuficiente & 1 & 1,3 & \\
\hline 31. Estase de secreções & 1 & 1,3 & \\
\hline $\begin{array}{l}\text { 32. Incapacidade para ingerir ou digerir comida ou absorver nutrientes causada por } \\
\text { fatores biológicos, psicológicos ou econômicos. }\end{array}$ & 1 & 1,3 & \\
\hline 33. Fator mecânico & 1 & 1,3 & P25 \\
\hline 34. Estrutura mamária normal & 1 & 1,3 & \\
\hline 35. Estrutura oral da criança normal & 1 & 1,3 & \\
\hline 36. Prejuízos músculo-esqueléticos, neuromusculares & 1 & 1,3 & \\
\hline 37. Percepção sensorial alterada & 1 & 1,3 & \\
\hline 38. Vias aéreas alérgicas & 1 & 1,3 & \\
\hline 39. Fumo passivo & 1 & 1,3 & \\
\hline 40. Doença ou trauma (hipertermia) & 1 & 1,3 & \\
\hline 41. Prejuízo/ retardo neurológico & 1 & 1,3 & \\
\hline 42. Umidade & 1 & 1,3 & \\
\hline 43. Falta de ar & 1 & 1,3 & \\
\hline
\end{tabular}

P25- Percentil 25; P50- Percentil 50; P75- Percentil 75.

Foram identificados 43 fatores relacionados, entre os quais, 10 encontravam-se acima do percentil 75 e estavam relacionados ao sistema respiratório, proteção, ambiente, nutrição e desempenho de papel. Destes, a maioria estavam presentes em uma proporção muito baixa das crianças com infecção respiratória aguda (IRA). Somente 4 fatores relacionados apresentaram freqüências superiores a $30 \%$ : Secreções retidas $(98,7 \%)$ e Hiperventilação (74,4\%), Perfis sanguíneos anormais (38,5\%), Barreiras ambientais (33,3\%) (Vide Tabela 3).

Tabela 4 - Distribuição dos fatores de risco presentes em crianças acometidas por infecção respiratória aguda. Fortaleza, 2005.

\begin{tabular}{|c|c|c|}
\hline Fatores de risco & $N^{\circ}$ & $\%$ \\
\hline 1. Procedimentos invasivos & 77 & 98,7 \\
\hline
\end{tabular}


2. Comportamentos de alimentação mal-adaptados, do indivíduo

3. Anorexia

4. Desnutrição

5. Perdas excessivas por vias normais

6. Hábitos irregulares de evacuação

7. Hábitos alimentares deficientes

8. Ingestão de fibras insuficiente

9. Fator mecânico

10. Esvaziamento gástrico retardado

11. Mudanças ambientais recentes

12. Agentes farmacêuticos

13. Defesas secundárias inadequadas

14. Mudança nos padrões habituais de comida e alimentação

$\begin{array}{ccc}38 & 48,7 & \\ 9 & 11,5 & \mathrm{P} 75 \\ 9 & 11,5 & \\ 5 & 6,4 & \\ 5 & 6,4 & \\ 3 & 3,8 & \mathrm{P} 50 \\ 3 & 3,8 & \\ 2 & 2,6 & \\ 2 & 2,6 & \\ 2 & 2,6 & \mathrm{P} 25 \\ 1 & 1,3 & \\ 1 & 1,3 & \\ 1 & 1,3 & \\ \end{array}$

P25- Percentil 25; P50- Percentil 50; P75- Percentil 75.

Do total de fatores de risco identificados, três apresentaram freqüências acima do percentil 75 e estavam relacionadas à infecção e à nutrição, indicando fatores de risco diretamente ligados aos problemas respiratórios apresentados pelas crianças em estudo. Os fatores de risco Procedimentos invasivos (98,7\%) e Comportamento de alimentação mal-adaptado do indivíduo (48,7\%) apresentaram freqüências elevadas (Vide Tabela 4).

\section{DISCUSSÃO}

Em unidades básicas de saúde, cerca de $40 \%$ das crianças apresentam algum tipo de problema de saúde, sendo os mais comuns, os problemas do aparelho respiratório (59,3\%), seguido dos problemas dermatológicos (33,3\%) e gastrintestinais $(7,4 \%)$ (SIQUEIRA, 2005).

Os dados do presente estudo permitiram a identificação de 26 diagnósticos de enfermagem nas crianças, entre os quais seis diagnósticos encontravamse acima do percentil 75: desobstrução ineficaz de vias aéreas, risco para infecção, padrão respiratório ineficaz, proteção ineficaz, risco para crescimento desproporcional, mucosa oral prejudicada. Os quatro últimos diagnósticos representaram fortes associações estatísticas entre si. Um estudo sobre diagnósticos de enfermagem em crianças com cardiopatias congênitas (SILVA, 2004) identificou diagnósticos de enfermagem semelhantes aos que foram encontrados neste estudo, num grande percentual. São eles: Padrão respiratório ineficaz (86,7\%), Risco para infecção $(82,2 \%)$ e Desobstrução ineficaz de vias aéreas $(55,6 \%)$.

Dois foram identificados em $100 \%$ das crianças portadoras de infecção respiratória aguda: Desobstrução ineficaz de vias aéreas e Risco para infecção. O fato do diagnóstico Desobstrução ineficaz de vias aéreas estar presente nas crianças com até cinco anos de idade portadoras de infecção respiratória aguda pode se relacionar ao diâmetro reduzido das vias aéreas nas crianças jovens, culminando com o estreitamento do lúmen devido ao edema das mucosas, produção exacerbada de secreções, infiltração de células inflamatórias levando, conseqüentemente, a graus variáveis de obstrução (WHALEY \& WONG, 1999).

O diagnóstico Desobstrução ineficaz das vias aéreas pode ser identificado em proporções diferentes na dependência do período de internamento. Em algumas situações cerca de $70 \%$ das crianças podem não apresentar este diagnóstico nas primeiras 48 horas de internação hospitalar. Mesmo em situações mais críticas, esse diagnóstico pode levar uma de média de 8 dias de internamento para ser identificado (SILVA, 2004).

O diagnóstico de enfermagem Risco para infecção é definido como estar em risco aumentado de ser invadido por organismos patogênicos (NANDA, 2002). Pode está relacionado à presença da patologia de base, a condições clínicas e a alguns fatores de risco, como procedimentos invasivos e à hospitalização, os quais predispõem a criança à manifestação de diversos tipos de infecção (SILVA, 2004).

Com relação ao diagnóstico Padrão Respiratório ineficaz, um estudo de validação deste diagnóstico em crianças hospitalizadas, indicou estreita associação com as infecções respiratórias agudas, principalmente com a presença da característica definidora "Dispnéia", a qual foi identificada com freqüência $98 \%$ e validada como importante achado dentre os sinais e sintomas das IRAs (SILVA, 1994). Esses dados foram semelhantes aos encontrados na presente investigação, que identificou freqüência de $74,4 \%$ da mesma característica definidora nas crianças acometidas por infecção respiratória aguda.

O diagnóstico de enfermagem Risco para crescimento desproporcional esteve presente em $48,7 \%$ das crianças deste estudo. Este diagnóstico pode ser representado pelos valores percentis acima de $97^{\circ}$ ou abaixo do $3^{\circ}$ percentil para idade que indicam risco nutricional (NANDA, 2002). Optou-se neste estudo por utilizar o escore $Z$ para representar a variabilidade peso/ altura entre as crianças. $O$ escore $Z$ representa a distância, em unidades de desvio padrão, obtido pela diferença entre o valor medido nos indivíduos e valor médio da população de referência, dividida pelo desvio padrão da mesma 
população (MS, 2002). De acordo com a classificação do Ministério da saúde: acima do percentil 97 ou escore $Z$ (entre 2 e 3): sobrepeso; entre os percentis 97 e 3 ou escore $Z$ (-2 a 2): faixa de normalidade nutricional; entre os percentis 10 e 3 ou escore $Z(-2$ e $-1 / 1$ e 2): risco nutricional para desnutrição ou sobrepeso; entre os percentis 3 e 0,1 ou escore $Z$ (-3 e -2): peso baixo para a idade; abaixo do percentil 0,1 ou escore Z ( abaixo de-3): peso baixo para idade (MS, 2002). Para tanto, obteve-se um valor médio do escore Z (peso/ altura) entre as crianças de $0,47( \pm$ 1,31 ), sendo que $25 \%$ das crianças apresentaram escore $Z$ até 0,41 , enquanto $75 \%$ até 1,46 . Portanto, as crianças se enquadraram entre os percentis 3 e 97 ou escore $Z$ (-2 a 2), correspondendo faixas de normalidade nutricional na análise dos parâmetros antropométricos. Em um estudo realizado com 58 crianças de 12 a 48 meses em Ribeirão Preto-SP, observa-se que $53,4 \%$ das crianças se encontram abaixo do $\mathrm{P} 10$, sendo que destas, $34,4 \%$ se encontram na faixa de normalidade, portanto valores similares ao nosso estudo (CARABOLANTE \& FERRIANI, 2003)

A presença do diagnóstico Proteção ineficaz em $38,5 \%$ das crianças pode relacionar-se a elevada freqüência dos perfis sangüíneos anormais identificada por exames laboratoriais ou, ainda, pelo fato do organismo infantil se apresentar extremamente fragilizado pelo acometimento das infecções. As deficiências do sistema imune colocam as crianças em risco para qualquer processo infeccioso e as taxas de infecção sobe dos 3 aos 6 meses de idade, no intervalo entre o desaparecimento dos anticorpos maternos e a produção de anticorpos pela crianças, fator este que colabora com a incidência de infecções no trato respiratório (WHALEY \& WONG, 1999).

$O$ diagnóstico de enfermagem Mucosa oral prejudicada foi o sexto mais comum dentre os diagnósticos que se encontravam acima do percentil 75. Sua alta freqüência pode estar relacionada ao fato das crianças com infecções respiratórias agudas apresentarem obstrução nasal, dor na garganta, que culminam com uso da respiração labial, além de se submeterem à antibioticoterapia, resultando em imunossupressão e, conseqüentemente, ao surgimento de placas brancas e distúrbios na mucosa oral. Alguns antibióticos, como os quelantes do cálcio, representados pelas tetraciclinas que são úteis nas infecções mistas das vias respiratórias, depositam-se nos ossos e dentes em crescimentos, causando manchas e, algumas vezes, hipoplasia dentária e deformidades ósseas (RANG et al, 2001). Ademais, a higiene oral ineficaz presente em $33,3 \%$ das crianças é outro fator contribuinte e revela influência dos fatores sócio-econômicos.

\section{CONCLUSÕES}

Verificou-se que a produção de estudos dos diagnósticos de enfermagem em crianças com infecção respiratória aguda ainda é muito escassa, o que mostra a necessidade de buscar outros estudos correlatos para subsidiar a discussão. Foram encontrados 26 diagnósticos de enfermagem, 43 fatores relacionados, 14 fatores de risco e 67 características definidoras diferentes. As crianças apresentaram, em média, 5,32 diagnósticos de enfermagem $( \pm 1,79)$. Seis diagnósticos apresentaram freqüências acima do percentil 75 , são eles: Desobstrução ineficaz de vias aéreas (100\%); Risco para infecção (100\%); Padrão respiratório ineficaz $(74,4 \%)$; Risco para crescimento desproporcional $(48,7 \%) ; \quad$ Proteção ineficaz (38,5\%); Membrana mucosa oral prejudicada $(34,6 \%)$. O perfil diagnóstico encontrado mostra grande prevalência de problemas relacionados à condição sócio-econômica das famílias das crianças, destacando-se as alterações respiratórias e ponderais. Considera-se que os dados apresentados contribuem para a implementação de planos de cuidados mais específicos e individualizados que atendam às peculiaridades do grupo etário em questão. Ademais, possibilitam o fortalecimento e expansão da prática do enfermeiro, auxiliando na interpretação e julgamento clínico dos problemas de saúde atuais ou potenciais baseados na evidência científica e na manifestação das respostas da criança.

\section{REFERÊNCIAS BIBLIOGRÁFICAS}

ANDRAUS, L.M.S. et al. Ensinando e aprendendo: uma experiência com grupos de pais de crianças hospitalizadas. Revista Eletrônica de Enfermagem [on line] 2004. Disponível em: http://www.fen.ufg.br/revista/revista6 1/pdf/r1 desvel a.pdf [Acesso em 24 jul. 2006]

BARATA, R.C.B. et al. Gastroenterites e infecções respiratórias agudas em crianças menores de cinco anos em área da região Sudeste do Brasil, 19861987. I - Infecções respiratórias agudas. São Paulo.Revista de Saúde Pública, v.30, n.6, p.553-63, 1996.

BOTELHO, C. et al. Fatores ambientais e hospitalizações em crianças menores de cinco anos com infecção respiratória aguda. Cadernos de Saúde Pública, v.19, n.6, p.1771 -1780, 2003.

BRASIL. Ministério da Saúde. Conselho Nacional de Saúde. Resolução 196. Decreto № 93.933 de janeiro de 1987. Estabelece critérios sobre pesquisa envolvendo seres humanos. Bioética, v.4, n.2, p.1525, 1996. Suplemento.

CAETANO, J.R.M. et al. Fatores associados à internação hospitalar de crianças menores de cinco anos, São Paulo, SP. Revista de Saúde Pública. v.36, n.3, p.285-91, 2002.

CARABOLANTE, A. C.; FERRIANI, M. G. C. O crescimento e desenvolvimento de crianças na faixa etária de 12 a 48 meses em creche na periferia da cidade de Ribeirão Preto - SP. Revista Eletrônica de Enfermagem [on line] 2003. Disponível em: 
http://www.fen.ufg.br/revista/revista5 1/cresci.html

[Acesso em 24 jul. 2006]

DUARTE, D.M.G.; BOTELHO, C. Perfil clínico de crianças menores de cinco anos com infecção respiratória aguda. Jornal de Pediatria. v.76, n.3, p.207-212, 2000.

FERREIRA, O.S.; BRITTO, M.C.A. Pneumonia aguda - tema que todos devemos estudar. Jornal de Pediatria. v.79, n.6, p.478, 2003.

GORDON, M. Nursing diagnosis: process and application. St. Louis: Mosby; 1994

LIRA, A.L.B.C. Diagnósticos de enfermagem em pacientes transplantados renais de um hospital universitário de Fortaleza-CE. [dissertação]. Fortaleza-(CE): Departamento de enfermagem/ UFC, 2005.

BRASIL. Ministério da saúde. Assistência e Controle das Infecções Respiratórias Agudas. 4aㅡ Ed.; Brasília: Coordenação Materno- Infantil, 1994.

BRASIL. Ministério da saúde. Secretaria de Políticas de Saúde. Departamento de Atenção Básica. Saúde da criança: acompanhamento do crescimento e desenvolvimento infantil. Brasília: Ministério da Saúde; 2002. p.28-33.

NORTH AMERICAN NURSING DIAGNOSIS ASSOCIATION (NANDA). Diagnósticos de enfermagem. Porto Alegre: Artmed, 2002.

PRIETSCH, S.O.M. et al. Doença aguda das vias aéreas inferiores em menores de cinco anos: infância do ambiente doméstico e do tabagismo materno. Jornal de Pediatria. v.78, n.5, p. 415-22, 2002.

RANG, H.P.; DALE, M.M.; RITTER, J.M. Farmacologia. 4ª edição, Rio de Janeiro:Guanabara Koogan, 2001.

RODRIGUES, O.G. et al. Infecções virais em crianças portadoras de doença respiratória aguda, atendidas em um Centro de Saúde Escola, em Belém, Pará, Brasil. Pediatria. v.26, n.1, p.13-20, 2004

SILVA, M.F.O. Validação do diagnóstico de enfermagem Padrão Respiratório Ineficaz à criança hospitalizada. [dissertação]. João Pessoa (PB): Centro de Ciências da Saúde/ UFPB, 1994.

SILVA, V.M. Crianças com cardiopatias congênitas: avaliação diagnóstica de enfermagem. [dissertação]. Fortaleza(CE): Departamento de enfermagem/UFC, 2004.

SIQUEIRA, R.C. Diagnósticos de enfermagem em crianças menores de um ano acompanhadas na consulta de enfermagem em puericultura numa unidade básica de saúde de Fortaleza-Ce. [dissertação]. Fortaleza(CE): Departamento de enfermagem/UFC, 2005.

WHALEY, L.F.; WONG, D.L. Enfermagem pediátrica: elementos essenciais à intervenção efetiva. 5aㅡ. ed., Rio de Janeiro: Guanabara Koogan, 1999. 